\title{
ENSEÑANZA DEL INGLÉS EN EL MÁSTER DE SECUNDARIA: LAS CREENCIAS DEL PROFESORADO EN FORMACIÓN INICIAL
}

\author{
Juana Herrera Cubas \\ Universidad de La Laguna
}

\section{RESUMEN}

Las creencias del profesorado en formación inicial sobre el aprendizaje de lenguas extranjeras están determinadas por las experiencias de aprendizaje propias y funcionan como filtros que pueden condicionar su formación y actuación profesional futura. Numerosos estudios concluyen que estas creencias son resistentes, pero se pueden cambiar. Este trabajo identifica cuatro aspectos de las prácticas externas del Máster de Formación del Profesorado de Secundaria que propiciaron ese cambio: la falta de competencia en la lengua extranjera de los estudiantes de secundaria, la escasa motivación por aprenderla, la metodología de aula observada y las propias experiencias de aprendizaje del profesorado en formación.

Palabras Clave: creencias del profesorado, formación inicial del profesorado, lenguas segundas y extranjeras.

\section{ENGLISH LANGUAGE TEACHING IN A MASTER'S \\ DEGREE OF SECONDARY EDUCATION: \\ THE BELIEFS OF PRE-SERVICE TEACHERS}

\section{Abstract}

Pre-service teachers' beliefs about foreign language learning are heavily influenced by their prior learning experience. These beliefs act as filters through which new knowledge is interpreted and teaching decisions in the professional context are taken. Numerous studies conclude that beliefs are resistant but can be changed. This paper presents four aspects of the practicum phase of the Masters' Degree of Secondary Education that actually contributed to change: secondary students' little competence in the foreign language; their lack of motivation to learn it; the teaching methodology observed and pre-service teachers' consciousness of their prior learning experience.

KEYWORDS: teachers' beliefs, pre-service teacher education, foreign and second languages. 


\section{INTRODUCCIÓN}

Numerosos estudios concluyen que los profesores en fase de formación inicial comienzan los cursos o programas formativos con ideas preconcebidas y bien arraigadas acerca de lo que constituye el proceso de enseñanza y aprendizaje, en cualquier ámbito del saber, fruto de sus experiencias propias y de su herencia cultural (Joram y Gabriel, 1998). Estas experiencias funcionan como filtros que pueden condicionar su modo de comprensión, e incluso aceptación, del conocimiento que reciben en los cursos de formación, y también sus creencias acerca de la práctica docente, creencias que, en muchos casos, permanecen inalteradas durante largo tiempo (Kagan, 19922a; Pajares, 1992).

En el ámbito de segundas lenguas y lenguas extranjeras, del mismo modo, las experiencias de aprendizaje previas conforman las creencias con las que los profesores acceden a los cursos de formación. Estas creencias, muchas veces erróneas e incoherentes con los resultados de la investigación en adquisición de segundas lenguas y la enseńanza de segundas lenguas y lenguas extranjeras, sirven como modelos para su práctica docente, tanto en la fase práctica de los programas de formación como en la práctica profesional subsiguiente.

El desarrollo profesional, sin embargo, demanda ir más allá de perpetuar estas conductas aprendidas y, por ello, es necesario que los programas de formación inicial, y especialmente la fase práctica en centros, proporcionen a los profesores oportunidades para reflexionar sobre sus creencias previas, para explorar las posibilidades de cambio cuando sea necesario y, al mismo tiempo, que les ofrezcan modelos de actuación adecuados y una dirección clara hacia la que avanzar (Johnson, 1994).

Las prácticas externas en centros, por lo tanto, lejos de ser un mero ejercicio de observación sistemática de la práctica de un tutor profesional o de las características y actividades propias del entorno educativo, constituyen un elemento clave en la formación, ya que las situaciones que surgen en el contexto de la práctica permiten a los profesores tomar consciencia de sus creencias, reflexionar sobre las mismas y tomar decisiones que tienen sentido porque responden a necesidades concretas de un contexto real; no son meros reflejos de experiencias de aprendizajes pasados.

El estudio que se presenta a continuación se llevó a cabo durante el curso académico 2016-2017 en la asignatura Prácticas de la especialidad de Enseñanza del inglés del Máster Interuniversitario en Formación del Profesorado de Educación Secundaria Obligatoria y Bachillerato, Formación Profesional y Enseñanza de Idiomas, ofertado por la Universidad de La Laguna. La finalidad de dicho estudio se concretó en tres objetivos bien definidos: a) identificar las creencias del profesorado en formación inicial acerca de la enseñanza y aprendizaje de lenguas extranjeras antes de realizar el periodo de prácticas externas en centros; b) comprobar si las respuestas acerca de esas creencias cambiaban al finalizarlo; c) identificar los motivos que justificaban dichos cambios, si los hubiera. 


\section{LAS CREENCIAS DEL PROFESORADO EN FORMACIÓN INICIAL}

La investigación sobre las creencias del profesorado en fase de formación inicial, en el ámbito de la enseńanza y aprendizaje del inglés como segunda lengua o lengua extranjera, se ha centrado en tres aspectos fundamentales (Borg, 2003): el papel de la experiencia de aprendizaje previa, la influencia de los programas o cursos de formación inicial y el impacto de las prácticas docentes en el desarrollo de dichas creencias. Se establece esta separación entre programas formativos y prácticas para distinguir los trabajos que investigan la evolución de las creencias a lo largo de la formación, sin referencia al contexto de la práctica, por un lado, y los que sí incluyen esa referencia, por otro.

El conocimiento personal experiencial que guía las actuaciones y creencias del profesorado en formación se ha descrito como «imágenes» por el carácter fuertemente visual y la naturaleza afectiva emocional de los recuerdos o memorias evocados en los relatos aportados en la investigación. Las «imágenes» constituyen, en definitiva, el conocimiento personal experiencial que conforma las creencias del profesorado en formación, y sirven, a la vez, como modelos para la acción (Calderhead y Robson, 1991).

Johnson (1994) examinó las creencias sobre la enseñanza y el aprendizaje del inglés como segunda lengua de un grupo de cuatro profesores que realizaban un periodo de prácticas de 15 semanas de duración, en un máster de enseñanza del inglés como segunda lengua. La finalidad del estudio era determinar el papel de dichas creencias en las decisiones que guiaban su práctica de aula y en las «imágenes» que transmitían de sí mismos como docentes de segundas lenguas.

A partir de los datos obtenidos de sus diarios de prácticas, las grabaciones de las clases impartidas, las reflexiones escritas de sus experiencias de aprendizaje propias y entrevistas personales, Johnson concluyó que el modelo dominante de acción de estos profesores se basaba en sus experiencias de aprendizaje previas e identificó cuatro «imágenes» fundamentales que daban forma a sus creencias sobre la enseñanza y aprendizaje de la lengua: las experiencias en el contexto formal (referencias a sus profesores, a las actividades que realizaban, a la organización de la clase...); las experiencias en el contexto informal (experiencias positivas de aprendizaje, uso de la lengua en contextos reales...); su propia «imagen» como futuros docentes (rechazo al modelo de enseñanza formal al que habían estado expuestos y al observado en su propia práctica); y sus percepciones acerca de los componentes del programa de formación (que juzgaron más o menos adecuados, o aceptaron en mayor o menor grado, en función de sus experiencias de aprendizaje formal e informal).

En un estudio posterior, Numrich (1996) investigó los aspectos de la práctica docente que resultaban de interés o creaban preocupación a un grupo de 26 profesores que cursaban un máster de enseñanza del inglés a estudiantes de otras lenguas. La información de los diarios de su práctica de aula, un análisis posterior de dichos diarios y una semblanza de sus experiencias previas de aprendizaje de lenguas permitieron identificar cuatro aspectos o temas de interés comunes a todos los participantes: los aspectos positivos y negativos de su práctica docente (ser creativos, tener 
buena comunicación con los alumnos...); los elementos que constituyen una enseñanza eficaz (atender a las demandas de los alumnos, contextualizar el aprendizaje dentro y fuera del aula...); las estrategias que permitirían mejorar los puntos débiles identificados en su práctica futura (controlar el tiempo de las actividades programadas, dar instrucciones comprensibles, formarse en diversos modelos de evaluación, centrar la enseńanza en los intereses de los alumnos...); y la transferencia o rechazo de contenidos en la programación de aula atendiendo a sus experiencias de aprendizaje positivas o negativas.

La transferencia resultó evidente en las explicaciones de un grupo de participantes que decidió integrar el componente cultural en la enseñanza de la lengua extranjera o diseñar tareas que implicaran un uso real de la lengua porque, en su propia experiencia como aprendices, estos aspectos habían resultado motivadores y positivos. El rechazo, igualmente, quedó claro al evitar los contenidos gramaticales y la corrección de errores en clase porque eran aspectos negativos o traumáticos de su experiencia de aprendizaje anterior.

La efectividad de los programas de formación como factor que contribuye al cambio de las creencias del profesorado; por otro lado, resulta un aspecto controvertido tanto en el ámbito de las lenguas extranjeras como en otros ajenos a este (Kagan, 1992a; 1992b), ya que los resultados de unos estudios concluyen que dichos programas no ejercen influencia o cambio alguno, mientras que otros afirman lo contrario.

En un estudio longitudinal de tres años de duración, Peacock (2001) afirma que las creencias de los participantes se mantuvieron estables a lo largo del programa que cursaban de formación en metodología para la enseñanza del inglés como lengua extranjera, y advierte del peligro que supone reproducir un modelo de enseñanza aprendizaje basado en concepciones inadecuadas o erróneas, heredadas del aprendizaje anterior.

En un marco formativo similar, Cabaroglu y Roberts (2000), sin embargo, concluyen que los programas sí tienen efecto sobre las creencias de los participantes y que, aunque los resultados variaron mucho de uno a otro, lo cierto es que, prácticamente, toda su muestra reflejó cambios. Señalan, igualmente, que esos cambios son siempre graduales y acumulativos, que los cursos de formación deben entenderse como una variable más - no un agente de cambio- en el desarrollo de las creencias de los profesores en formación y que el factor experiencial, en el sentido de realizar actividades prácticas, resulta definitivo para tomar consciencia de la evolución de las creencias propias.

Las conclusiones de Busch (2010) apuntan en la misma dirección, ya que la experiencia de sus candidatos de tutelar a un alumno como parte de las actividades del programa de máster en adquisición de segundas lenguas que realizaban les permitió examinar sus propias creencias y establecer conexiones entre estas, los contenidos del curso y el aprendizaje de la lengua en un contexto real. Mattheoudakis (2007), además, aclara que los cursos son más efectivos cuando combinan teoría y práctica porque los planteamientos exclusivamente teóricos suelen resultar demasiado abstractos. Igualmente, señala que la realidad, muchas veces conflictiva, de la práctica de aula ejerce una influencia decisiva sobre los participantes que, en su 
estudio, mostraron actitudes más conservadoras en sus creencias al finalizar dicha práctica. Estos resultados coinciden con los aportados por Özmen (2012) respecto a la importancia de combinar teoría y práctica, y los de Wong (2010) sobre el papel de las nuevas experiencias de aprendizaje y los cambios de actitud.

Debreli (2016), también, destaca la dificultad de los participantes en su estudio para llevar a la práctica los conocimientos teóricos que informaban sus creencias y explica que, una vez advirtieron que los enfoques y técnicas aprendidos no siempre eran eficaces en el contexto real, respondieron de manera flexible, en unos casos, adaptando ese conocimiento a la realidad del aula, mientras que en otros demostraron poca capacidad de reflexión y adaptación, recurriendo a métodos y enfoques más tradicionales fruto, seguramente, de su propia experiencia de aprendizaje anterior. Concluye, por lo tanto, que las creencias iniciales son ajenas a la práctica, es decir, se conforman independientemente de la práctica, y que el conocimiento teórico no es suficiente para resolver situaciones propias del aula que demandan un repertorio amplio de técnicas de enseñanza y capacidad de adaptación.

El concepto de "cambio», continúa Debreli (2016), es más complejo de lo que pueda parecer, ya que hay una interacción constante entre las creencias iniciales y lo observado o experimentado en la práctica, que lleva a reconsiderar, cambiar o consolidar esas creencias. Yuan y Lee (2014) llegan a la misma conclusión y añaden, además, tres factores que facilitan el proceso de reflexión y cambio en el contexto profesional: la interacción con el tutor, los alumnos y otros miembros de la comunidad educativa; las tareas a realizar y, por último, las dificultades que van surgiendo de la propia práctica.

Evidentemente, existe una relación recíproca entre las creencias con las que el profesorado comienza su formación y el proceso de reflexión consciente sobre las mismas que tiene lugar en la práctica y que, a la vez, impulsa el cambio para la mejora de dicha práctica y del propio sistema de creencias. Los cambios son lentos y graduales y pueden, en ocasiones, afectar a una parte mínima de las creencias (Çapan, 2014) pero, en cualquier caso, lo cierto es que la mayoría de estudios coincide en que el contexto de la práctica es fundamental y decisivo para el desarrollo profesional.

\section{DESCRIPCIÓN DEL ESTUDIO}

El estudio que se presenta a continuación se desarrolló en la asignatura Prácticas del Máster Interuniversitario en Formación del Profesorado de Educación Secundaria Obligatoria y Bachillerato, Formación Profesional y Enseñanza de Idiomas por las Universidades de La Laguna y Las Palmas de Gran Canaria ${ }^{1}$ (en ade-

${ }^{1}$ Máster Interuniversitario en Formación del Profesorado de Educación Secundaria Obligatoria y Bachillerato, Formación Profesional y Enseñanzas de Idiomas por las Universidades de La Laguna y Las Palmas de Gran Canaria. Habilita para el ejercicio de actividades profesionales según la Orden EDU/3498/2011, de 16 de diciembre $-B O E$ n. ${ }^{\circ} 310$, de 26 de diciembre de 2011-, por la que se modifica la Orden ECI/3858/2007, de 27 de diciembre $-B O E$ n. ${ }^{\circ} 312$, de 29 de diciembre de 
lante, Máster de Secundaria). Puesto que el contexto de este trabajo se sitúa en la primera universidad, se hará mención al máster conforme a la oferta que se realiza desde la misma y, más concretamente, a la especialidad de Enseñanza del inglés, que es la que aquí compete.

\subsection{Participantes}

El número de participantes matriculados en la especialidad de Enseńanza del inglés durante el curso 2016-2017 sumó un total de 22, procedentes, principalmente, del Grado de Estudios Ingleses (54,54\%), la anterior licenciatura en Filología inglesa y alemana (18,18\%) y el Grado de Traductores e Intérpretes (27,27\%).

Puesto que los créditos de esta materia están repartidos al cincuenta por ciento entre los departamentos de Didácticas Específicas -Facultad de Educacióny Filología Inglesa y Alemana -Facultad de Humanidades, Sección de Filología-, el total de los 22 participantes se dividió en dos partes iguales y solo los asignados al primer departamento se incluyeron en este estudio. La muestra final, entonces, quedó compuesta por ocho mujeres y tres hombres, con edades comprendidas entre $\operatorname{los} 24$ y los 30 años.

\subsection{Contexto}

El Máster de Secundaria ofertado por la Universidad de La Laguna cuenta con 15 especialidades que responden a las especializaciones docentes implantadas en la Comunidad de Canarias. Este máster comprende un plan de estudios de 60 créditos de duración y tres módulos con sus correspondientes competencias: un Módulo Genérico de 15 créditos, con contenidos relativos a cuatro campos científicos y sus respectivas áreas de conocimiento (Psicología Evolutiva y de la Educación, Didáctica y Organización Escolar, Sociología de la Educación y Teoría e Historia de la Educación); un Módulo Específico de 24 créditos, con contenidos propios de las didácticas específicas de cada especialidad; y un Módulo Practicum de 21 créditos, con contenidos, igualmente, de las didácticas general y disciplinares, fundamentalmente, y de los campos de la formación profesional y la orientación psicopedagógica.

En la especialidad que nos ocupa, la asignatura Prácticas, incluida en el Módulo Practicum, debe proporcionar al estudiante cuatro competencias específicas que resulta de interés destacar: a) adquirir experiencia en la planificación de la docencia y la evaluación de las materias correspondientes a la especialización; b) acreditar un buen dominio de la expresión oral y escrita de la práctica docente; c)

2007-, por la que se establecen los requisitos para la verificación de los títulos universitarios oficiales que habiliten para el ejercicio de las profesiones de Profesor de Educación Secundaria Obligatoria y Bachillerato, Formación Profesional y Enseñanzas de Idiomas. 
dominar las destrezas y habilidades sociales necesarias para fomentar un clima que facilite el aprendizaje y la convivencia; y d) participar en las propuestas de mejora en los distintos ámbitos de aplicación a partir de la reflexión basada en la práctica.

Para la realización de estas prácticas, la Consejería de Educación, Universidades y Sostenibilidad del Gobierno de Canarias pone a disposición de las universidades su Red de centros de educación secundaria. Los centros tienen que contar con la totalidad de cursos que comprende la ESO, indicar las actividades formativas y organizativas que se comprometen a desarrollar, a partir de una propuesta elaborada a tal fin, designar profesorado tutor y coordinador y autorizar, de forma expresa, la presencia y participación del alumnado en prácticas en dichas actividades ${ }^{2}$.

En cuanto a los aspectos formativos, la duración de esta asignatura es de ocho semanas, con un total de 300 horas, equivalentes a 12 créditos, que se distribuyen del modo siguiente: 100 horas de trabajo autónomo; cinco de actividades de evaluación; y 195 de actividades presenciales que requieren seguimiento del docente. De estas, 175 se destinan al periodo de permanencia en los centros, y 20 a seminarios de preparación y seguimiento.

Durante el periodo de formación en los centros, los alumnos deben llevar a cabo su Programa individual de prácticas ${ }^{3}$, que comprende actividades y tareas tales como clases ordinarias en la ESO, Bachillerato o Formación Profesional; clases de apoyo al alumnado con materias pendientes; tutorías de grupo en la ESO y Bachillerato; programas específicos (TIC, Biblioteca escolar, CLIL...); actividades extraescolares y complementarias, de coordinación didáctica, con el equipo directivo y con el departamento de orientación; guardias o vigilancias; reuniones de departamento; atención tutorial a familias y al alumnado; reuniones del claustro, de los equipos docentes, de evaluación...

El seguimiento del programa de prácticas por parte del tutor universitario, por último, se lleva a cabo mediante seminarios durante los cuales se debe comprobar el desarrollo y cumplimiento del programa, asesorar y atender las consultas o

2 La propuesta general de actividades, acordada por las dos universidades canarias y los representantes de la administración educativa autonómica durante el proceso de elaboración del título original (Orden ECI/3858/2007, de 16 de diciembre), se encuentra publicada en la Resolución de 14 de diciembre de 2010 de la Dirección General de Ordenación, Innovación y Promoción Educativa -Anexo III-, Folios14-15. Esta propuesta de actividades original se mantiene, como tal, en el nuevo máster (Orden EDU/3498/2011, de 27 de diciembre).

${ }^{3}$ El Programa individual de prácticas, que todos los cursos se recoge como Anexo 2 en el Documento de apoyo a la e-guía de prácticas (e-guía: https://e-guia.ull.es/educacion/query.php?codigo $=125771015)$, comprende el conjunto de actividades formativas y organizativas ofertadas por cada centro, a partir de la propuesta general de actividades mencionada en la nota anterior. Si bien la elaboración de este programa individual debe ser una responsabilidad compartida entre los tutores de centro y universidad, para este estudio se preparó una propuesta alternativa, consistente en la inclusión de todas las actividades consideradas básicas y fundamentales para la formación de un docente, con un total de 175 horas, y las actividades específicas propias de cada centro, con 20 horas más, a distribuir por los tutores. La propuesta final contó 195 horas, en lugar de 175, que todos los tutores y centros aceptaron. 
dudas de los alumnos y promover la reflexión sobre la práctica docente y el intercambio de experiencias entre los compañeros.

\subsection{INSTRUMENTOS PARA LA RECOGIDA DE DATOS Y PROCEDIMIENTO}

Para la recogida de datos se utilizaron dos instrumentos fundamentales: el cuestionario Beliefs About Language Learning Inventory (BALLI) (Horwitz, 1988), modificado para este estudio, y los informes semanales de las prácticas, que forman parte la memoria final de la asignatura.

\subsubsection{El cuestionario BALLI}

El cuestionario BALLI, diseñado por Elaine Horwitz para identificar y establecer sistemas de creencias de profesores y estudiantes acerca del aprendizaje de las lenguas más comúnmente estudiadas en EE. UU. -inglés, español, alemán y francés-, es el instrumento más utilizado desde la década de los 90, que marca la consolidación de este modelo de investigación, hasta hoy. A lo largo de sus tres versiones (Kuntz, 1996a) -la primera destinada a estudiantes de segundas lenguas (Horwitz, 1987); una segunda a profesores de lenguas extranjeras (Horwitz, 1985); y la tercera a estudiantes de lenguas extranjeras (Horwitz, 1988) - el BALLI ha aumentado hasta 34 el número de ítems a los que profesores y estudiantes deben responder acerca de sus creencias, conforme a las cinco opciones de la escala Likert: 1 (muy de acuerdo), 2 (de acuerdo), 3 (ni de acuerdo ni en desacuerdo/neutral), 4 (en desacuerdo) y 5 (muy en desacuerdo).

Los 34 ítems de la versión de 1988 se agrupan en cinco ámbitos ${ }^{4}$ diferentes, fácilmente identificables en el aprendizaje de lenguas extranjeras: Dificultad de la lengua (Language difficulty), Aptitud para el aprendizaje de lenguas extranjeras (Foreign language aptitude), Características del aprendizaje de las lenguas (Nature of language learning), Estrategias para el aprendizaje y la comunicación (Learning and communication strategies) y Motivación y expectativas (Motivation and expectations).

Si bien la validez del instrumento ha sido cuestionada (Kuntz, 1996a) por diversos motivos -cambios no justificados en el orden de los ítems, organización no razonada de los ámbitos o los ítems que los componen e, incluso, la propia estructura del cuestionario5, especialmente en la versión de 1985-, lo cierto es que el BALLI se ha utilizado en, prácticamente, la mayoría de estudios sobre las creencias de profe-

${ }^{4}$ Horwitz utiliza el término themes (temas) para nombrar los cinco grupos de ítems que conforman el BALLI. En este estudio se ha optado por el término «ámbito», ya que el sentido de la palabra inglesa no se corresponde, exactamente, con su equivalente español.

${ }_{5}$ Kuntz (1996a) argumenta que los estudios de Horwitz estaban enfocados en identificar las creencias de los estudiantes sobre el aprendizaje de lenguas. Los ítems que conforman los BALLI, sin embargo, no se generaron a partir de las respuestas de los estudiantes, sino de las opiniones de los 
sores y alumnos en el área de lenguas (Borg, 2003; Gao, 2014; Zheng, 2009), que los resultados de estos estudios, además, coinciden, y que el patrón de ámbitos establecido y los ítems que los integran muestran un grado de cohesión razonable, factores todos que permiten concluir que el cuestionario BALLI es un instrumento adecuado y válido para investigar el sistema de creencias sobre el aprendizaje de lenguas de profesores y estudiantes en distintos contextos sociolingüísticos y socioculturales (Nikitina \& Furuoka, 2006).

Este último aspecto de adaptación al contexto sociolingüístico y sociocultural justifica las modificaciones realizadas en la versión del BALLI de 1988 utilizada en este estudio, modificaciones consistentes, principalmente, en la eliminación de tres ítems originales, una reagrupación de cuatro ítems respecto a sus ámbitos iniciales y la adición de siete nuevos ítems que reflejan opiniones e ideas comunes acerca del aprendizaje de lenguas (Lightbown \& Spada, 2011). El cuestionario final, por tanto, constó de 38 ítems.

\subsubsection{Informes semanales de las prácticas}

Además del cuestionario BALLI, se utilizaron los informes semanales de las actividades realizadas en los centros de prácticas durante las ocho semanas de duración de las mismas. Se hizo hincapié, a lo largo de los seminarios de seguimiento, en la importancia de elaborar informes reflexivos y analíticos, frente a los meramente descriptivos, de la práctica de aula observada y, especialmente, de la propia, ya que examinar de forma crítica nuestras actuaciones y principios acerca de la práctica, un proceso de aprendizaje que tiene lugar en y desde la experiencia, supone un paso cualitativo en la dirección de entender y mejorar esa práctica (Finlay, 2008).

Formar profesionales reflexivos, capaces de resolver problemas, tomar decisiones y proponer soluciones a partir de la reflexión sobre la práctica, como propone este máster, implica, necesariamente, experimentar, observar y reflexionar sobre la práctica propia, ya que solo de este modo, y no mediante la descripción de las experiencias ajenas, se podrá hacer de la práctica profesional una realidad propia. La reflexión, por tanto, debe servir para guiar el proceso de formación en la práctica, no para finalizarlo.

profesores acerca de cuáles serían las creencias de estos estudiantes. Los ámbitos, por tanto, no representarían estructuras de las creencias de los estudiantes, propiamente, según esta autora. 


\subsection{Procedimiento}

A partir de las pautas establecidas en la e-guía docente de la asignatura, se celebraron dos seminarios preparatorios previos a la incorporación de los participantes a los centros, cuatro seminarios de seguimiento y uno final, además de una entrevista individual con cada uno.

Puesto que la finalidad de este estudio consistía en comprobar si las respuestas de los participantes cambiaban antes y después del periodo de prácticas, se administró dos veces el cuestionario BALLI, la primera de ellas antes de la incorporación a los centros, durante el primer seminario -primera vuelta-, y la segunda al terminar las prácticas, en el seminario final -segunda vuelta-. Además, se solicitó una tarea escrita consistente en comentar algunos de los ítems que mostraban cambios importantes.

Cada semana, igualmente, se analizaron los informes de las actividades realizadas dentro y fuera de las aulas, a fin de contar con información adicional que permitiera, en primer lugar, realizar inferencias más precisas acerca de las creencias de los participantes sobre el aprendizaje de lenguas, más allá de los datos aportados en las respuestas al cuestionario (Pajares, 1992), y, en segundo lugar, identificar los motivos que justificaran los cambios, si los hubiera, en dichas respuestas.

\section{ANÁLISIS DE LOS DATOS Y RESULTADOS}

Para el análisis de los datos de este estudio se utilizó la prueba de rangos con signo de Wilcoxon para comparar las medias y el programa estadístico SPSS versión 21. En primer lugar, se volcaron las respuestas de los participantes a los 38 ítems del BALLI antes y después de la realización de las prácticas y, a continuación, se llevó a cabo el cálculo de las medias de ambas respuestas. En las tablas que se incluyen a continuación, se presenta el único ítem -número 12-cuyas respuestas variaron de forma estadísticamente significativa al final de las prácticas, con un valor $p<0.05$ (tabla 1), y un número de ítems que, si bien no reflejan cambios de significatividad estadística, con un valor $p>0.05$ (tablas 2, 3, 4), merecen ser analizados, ya que aportan una visión de la evolución de las respuestas de los participantes que resulta de interés. En todos los casos, los ítems se agrupan en sus ámbitos correspondientes ${ }^{6}$.

${ }^{6}$ La convención que se sigue en este estudio con respecto a las palabras extranjeras es el uso de la cursiva. En las tablas, sin embargo, se ha optado por las comillas («...») ya que la cursiva dificulta, considerablemente, la lectura. 


\begin{tabular}{|c|c|c|c|c|}
\hline \multicolumn{5}{|c|}{$\begin{array}{c}\text { TABLA 1. ÁMBITO MOTIVACIÓN Y EXPECTATIVAS. RESPUESTAS ANTES -PRE- } \\
\text { Y DESPUÉS -POST- DE LAS PRÁCTICAS, MEDIAS, DIFERENCIA MEDIAS (Dif.) } \\
\text { Y DESVIACIÓN TÍPICA (DT) * P }<0.05\end{array}$} \\
\hline \multirow{2}{*}{ PVALOR } & \multirow{2}{*}{ ÁMBITO MOTIVACIÓN Y EXPECTATIVAS } & \multicolumn{3}{|c|}{ Media (DT) } \\
\hline & & PRE & POST & Dif. \\
\hline$p=0.527$ & $\begin{array}{l}\text { 11. «If learners get to speak English very well, they will have } \\
\text { many opportunities to use it». }\end{array}$ & $\begin{array}{c}2.73 \\
(0.905)\end{array}$ & $\begin{array}{c}2.91 \\
(1.136)\end{array}$ & 0.18 \\
\hline$p=0.015$ & $\begin{array}{l}\text { 12. «I believe learners will ultimately learn to speak English } \\
\text { very well». }\end{array}$ & $\begin{array}{c}3.00 \\
(0.447)\end{array}$ & $\begin{array}{c}3.91 \\
(0.944)\end{array}$ & $0.91^{*}$ \\
\hline
\end{tabular}

\begin{tabular}{|c|c|c|c|c|}
\hline \multicolumn{5}{|c|}{$\begin{array}{l}\text { TABLA 2. ÁMBITO DIFICULTAD DE LA LENGUA. RESPUESTAS ANTES -PRE- } \\
\text { Y DESPUÉS -POST- DE LAS PRÁCTICAS, MEDIAS, DIFERENCIA MEDIAS (Dif.) } \\
\text { Y DESVIACIÓN TÍPICA (DT) }\end{array}$} \\
\hline \multirow{2}{*}{ PVALOR } & \multirow{2}{*}{ Ámbito DificulTAD DE LA LENGUA } & \multicolumn{3}{|c|}{ Media (DT) } \\
\hline & & PRE & POST & Dif. \\
\hline$p=0.630$ & 4. «Some languages are easier to learn than others». & $\begin{array}{c}1.64 \\
(0.674)\end{array}$ & $\begin{array}{c}2.18 \\
(0.982)\end{array}$ & 0.54 \\
\hline$p=0.132$ & $\begin{array}{l}\text { 25. «It is easier to read and write a foreign language than to } \\
\text { speak and understand it». }\end{array}$ & $\begin{array}{c}2.55 \\
(0.820)\end{array}$ & $\begin{array}{c}3.00 \\
(0.894)\end{array}$ & 0.45 \\
\hline
\end{tabular}

\begin{tabular}{|c|c|c|c|c|}
\hline \multicolumn{5}{|c|}{$\begin{array}{l}\text { TABLA 3. ÁMBITO CARACTERÍSTICAS DEL APRENDIZAJE DE LAS LENGUAS. } \\
\text { RESPUESTAS ANTES -PRE-Y DESPUÉS -POST-DE LAS PRÁCTICAS, MEDIAS, } \\
\text { DIFERENCIA MEDIAS (Dif.) Y DESVIACIÓN TÍPICA (DT) }\end{array}$} \\
\hline \multirow{2}{*}{ PVALOR } & \multirow{2}{*}{$\begin{array}{l}\text { ÁmBito CARACTERístiCAS DEL APRENDIZAJE } \\
\text { DE LAS LENGUAS }\end{array}$} & \multicolumn{3}{|c|}{ Media (DT) } \\
\hline & & PRE & POST & Dif. \\
\hline$p=0.791$ & $\begin{array}{l}\text { 8. «The earlier a language is introduced in school programs, } \\
\text { the greater the likelihood of success in learning». }\end{array}$ & $\begin{array}{c}2.18 \\
(1.079)\end{array}$ & $\begin{array}{c}2.27 \\
(1.009)\end{array}$ & 0.09 \\
\hline$D=0.194$ & $\begin{array}{l}\text { 18. «Learning a foreign language is mostly a matter of learning } \\
\text { a lot of new vocabulary words». }\end{array}$ & $\begin{array}{c}3.55 \\
(1.036)\end{array}$ & $\begin{array}{c}3.91 \\
(0.302) \\
\end{array}$ & 0.36 \\
\hline 1.000 & $\begin{array}{l}\text { 27. «Learning a foreign language is different form learning } \\
\text { other school subjects». }\end{array}$ & $\begin{array}{c}2.55 \\
(0.934)\end{array}$ & $\begin{array}{c}2.45 \\
(0.934)\end{array}$ & 0.10 \\
\hline
\end{tabular}

\begin{tabular}{ccccc}
\hline \multicolumn{4}{c}{ TABLA 4. ÁMBITO ESTRATEGIAS PARA EL APRENDIZAJE Y LA COMUNICACIÓN. } \\
RESPUESTAS ANTES -PRE-Y DESPUÉS -POST-DE LAS PRÁCTICAS, MEDIAS, \\
DIFERENCIA MEDIAS (Dif.) Y DESVIACIÓN TÍPICA (DT)
\end{tabular}

Como se puede observar en los resultados presentados en las tablas y gráficos, cuatro de los cinco ámbitos del BALLI registran cambios que serán analizados en las páginas siguientes: Motivación y expectativas (dos ítems), Dificultad de la lengua (dos ítems), Características del aprendizaje de las lenguas (tres ítems) y 
Estrategias para el aprendizaje y la comunicación (un ítem). El ámbito Aptitud para el aprendizaje de lenguas extranjeras no revela cambio alguno.

De igual modo, las respuestas del conjunto de los participantes, antes y después de la realización de las prácticas en los centros, muestran cambios con medias que oscilan entre 1.55-4.83, en la primera vuelta, y 1.55-4.91, en la segunda, con valores $\mathrm{p}>0.05$. Estos cambios no se consideran significativos desde el punto de vista estadístico de las medias, pero son cambios, en sentido estricto, ya que indican movimientos hacia uno u otro lado de la escala que reflejan, en definitiva, diferencias de opinión de los participantes. El único cambio que sí posee significatividad se refiere al ítem 12 , con valor $\mathrm{p}=0.015$ y medias de 3.00 y 3.91 .

\subsection{Discusión De los RESUltados}

Los motivos que justifican los cambios en las respuestas de los participantes al final del periodo de prácticas, tal como recogen los informes semanales y las opiniones expresadas en los seminarios de seguimiento, se pueden resumir en cuatro: el escaso conocimiento de la lengua extranjera de los alumnos de secundaria; la falta de motivación e interés por aprenderla; el modelo metodológico observado en las aulas; y la toma de conciencia de las experiencias de aprendizaje de los propios participantes en este estudio. Estos cuatro aspectos, como veremos a continuación, ejercieron una influencia definitiva sobre las creencias con las que iniciaron el periodo de prácticas.

Como se puede observar, en la tabla 1, correspondiente al ámbito Motivación y expectativas, se produce un aumento en el número de participantes en desacuerdo con la afirmación de que los estudiantes que aprendan inglés tendrán muchas oportunidades de usarlo (ítem 11, medias pre 2.73 y post 2.91 ). Este cambio se produce, principalmente, por el número de respuestas neutrales -ni de acuerdo ni en desacuerdo- que se suma a los desacuerdos $(27,27 \%)$ en la segunda vuelta.

Las repuestas al ítem 12 (medias pre 3.00 y post 3.91) están en consonancia con las del anterior, pues indican, claramente, un aumento de los participantes que no creen que todos los estudiantes aprenderán, al final, a hablar inglés muy bien. La diferencia con respecto al primero, sin embargo, resulta evidente si consideramos que, en este caso, hay un $45,45 \%$ de respuestas neutrales que pasan a desacuerdo $(18,18 \%)$ o muy en desacuerdo $(27,27 \%)$ en la segunda vuelta; se registra, también, un participante que estaba en desacuerdo y, ahora, muy en desacuerdo con esta afirmación. Más de la mitad de la muestra (54,54\%), entonces, se ha inclinado significativamente $(p=0.015)$ en una misma dirección.

Tal como reflejan los informes semanales de las prácticas, los motivos que justifican estos cambios en las respuestas son, principalmente, el desconcierto de los participantes ante el escaso conocimiento de la lengua inglesa de sus alumnos de secundaria y, peor aún, la falta de motivación e interés por aprenderla. Conforme a sus valoraciones, la mayoría de los adolescentes apenas alcanza el A2 del Marco Común Europeo de Referencia para las Lenguas (MCERL) en las destrezas de comprensión, producción e interacción oral y escrita, y en esta última está más cerca del A1 en la interacción oral. 
Por otro lado, señalan, resulta muy contradictorio que reconozcan, al menos verbalmente, la importancia del inglés en el momento actual, principalmente en el plano profesional, y que luego sean incapaces de ver la utilidad y las ventajas que su aprendizaje puede reportarles en el mundo real. Esto, aclaran, podría justificar la falta de motivación y compromiso a la hora de realizar las tareas que les proponían dentro y, sobre todo, fuera del aula. No sorprende, por lo tanto, que haya un cambio de opinión e, incluso, un cierto pesimismo (Kagan, 1992b; Mattheoudakis, 2007) de los participantes con respecto a la posibilidad de que todos los alumnos logren, al final, hablar bien la lengua extranjera y tengan, en consecuencia, oportunidades de usarla.

En la tabla 2, Dificultad de la lengua, el 90,90\% de la muestra estaba de acuerdo o muy de acuerdo, inicialmente, en que unas lenguas son más fáciles de aprender que otras (ítem 4, medias pre 1.64 y post 2.18); sin embargo, aunque sus respuestas en esta segunda vuelta siguen siendo, mayoritariamente, a favor de la afirmación (63,63\%), se aprecia un aumento de indecisos (27,27\%) e, incluso, algún desacuerdo $(9,09 \%)$.

En el ítem 25 (medias pre 2.55 y post 3.00), por otro lado, se observa una dispersión en las respuestas de los participantes, ya que aumentan, claramente, las que indican no estar de acuerdo en que leer y escribir una lengua extranjera es más fácil que hablarla y entenderla oralmente $(36,36 \%)$, pero sigue habiendo un número considerable de opiniones a favor de la afirmación $(36,36 \%)$ o neutrales $(27,27 \%)$.

Claramente, los motivos mencionados en el ámbito de motivación son, en buena parte, los mismos que explican los cambios relacionados con la dificultad de la lengua. Sin embargo, los informes revelan otros dos que aportan datos de interés: las experiencias de aprendizaje de los propios participantes y el modelo metodológico usado en las aulas. Prácticamente la totalidad de la muestra respondió, inicialmente, que algunas lenguas son más fáciles de aprender que otras tomando como referencia, según argumentaron, su propia experiencia de aprendizaje del inglés en secundaria y de otras lenguas que estudian actualmente, como el alemán o el francés, que consideran más difíciles por la complejidad gramatical, fundamentalmente.

Sin embargo, a partir de lo observado sobre los niveles de competencia de los alumnos de secundaria, una parte importante reconsideró su opinión. El siguiente testimonio lo ilustra acertadamente: "Ahora que veo mis respuestas en las dos vueltas del cuestionario, estoy convencida de que en la primera respondí pensando, únicamente, en mi experiencia personal; sin embargo, estas prácticas me han servido para comprobar que, tal vez, no sea muy sensato entrar en una clase con ideas preconcebidas sobre lo que puede resultar fácil o difícil para cada uno, no digamos ya sobre la profesión».

Por otro lado, si se tiene en cuenta que las valoraciones sobre el nivel de competencia de los alumnos de secundaria señalan serias deficiencias en las destrezas de comprensión y producción oral y escrita y, especialmente, en la de interacción oral, no sorprende que una parte importante de la muestra se reafirme en la idea de que hablar y entender es más difícil que leer y escribir, mientras que otra considera que no se puede hacer tal distinción, es decir, que comprensión, producción e interacción son igualmente difíciles para los alumnos. 
La opinión generalizada a este respecto es que la metodología usada en el aula tiene más influencia en la adquisición y desarrollo de las destrezas que la dificultad intrínseca de cada una de ellas, es decir, si no se trabaja la lengua como un instrumento de comunicación cuya utilidad trasciende el ámbito escolar, y se proporciona la práctica necesaria de todas las destrezas, con la metodología adecuada, no se facilitará el desarrollo de ninguna ni, en suma, el interés por el aprendizaje de la lengua.

Los siguientes tres cambios de interés se producen en el ámbito Características del aprendizaje de las lenguas, tabla 3, con una tendencia hacia el aumento de respuestas neutrales y en desacuerdo con las afirmaciones presentadas. Así, el 72,72\% de los participantes, que opinaba que las probabilidades de éxito en el aprendizaje de una lengua aumentan si esa lengua se introduce temprano en los programas escolares (ítem 8 , medias pre 2.18 y post 2.27), no tiene ahora una opinión tan clara al respecto, con lo que disminuye el número de acuerdos en favor de las respuestas neutrales (27.27\%). Algo similar sucede con los que pensaban que las lenguas extranjeras no se aprenden como el resto de asignaturas (ítem 27, medias pre 2.55 y post 2.45 ), ya que del $54,54 \%$ inicial, un $27,27 \%$ duda ahora de esta afirmación.

En cuanto al tercer cambio (ítem 18 , medias pre 3.55 y post 3.91 ), el $72,72 \%$ consideraba que aprender una lengua extranjera no se reduce a conocer mucho vocabulario, opinión que se generaliza hasta un $90,90 \%$, con el $18,18 \%$ de cambios procedentes de los que opinaban que esto era así.

De nuevo, la escasa competencia en la lengua de los adolescentes y la metodología de enseñanza son factores que explican los cambios de opinión. Si bien es muy cierto que el aprendizaje temprano puede ser un factor de éxito, señalan como dato significativo el número de años que los escolares de nuestro país dedican al estudio del inglés y los resultados poco satisfactorios que obtienen. En este sentido, las opiniones de muchos de los participantes coinciden en que perpetuar un modelo estructuralista y tradicional basado, como su nombre indica, en la descripción de estructuras gramaticales que siguen el orden establecido por las casas editoriales y que, además, carecen, en la mayoría de los casos, de aplicación práctica más allá de ejercicios convencionales; o fomentar el aprendizaje memorístico, frente al estratégico, de listas de palabras descontextualizadas, son factores que no contribuyen a transmitir a los aprendices el valor auténtico de la lengua extranjera como un instrumento para la comunicación y la adquisición de otros conocimientos, necesario y fundamental en el mundo real y en la sociedad globalizada en que vivimos.

El proceso de aprendizaje de una lengua extranjera es lento y requiere tiempo y dedicación y, a diferencia del resto de asignaturas del currículo, mucha práctica significativa en contextos de uso real. No parece, por lo tanto, a juicio de un número importante de participantes que el éxito en el aprendizaje de la lengua esté vinculado solo, o principalmente, a la edad con que se inicie en el sistema educativo, sino, en gran medida y entre otros muchos factores, a la metodología y a las condiciones en que se realice ese proceso de enseńanza aprendizaje. La propia palabra "proceso» implica ya una duración que no puede limitarse a cuatro horas semanales ni al estudio de los aspectos formales de la lengua, únicamente. 
El último cambio de interés, en el ámbito Estrategias para el aprendizaje y la comunicación (tabla 4), se refiere a los participantes que, inicialmente, no tenían una opinión definida $(72,72 \%)$ acerca de la importancia de practicar la lengua en un laboratorio de idiomas (ítem 35, medias pre 2.91 y post 3.18), la mitad de los cuales se decanta, ahora, hacia el desacuerdo o muy en desacuerdo con la afirmación $(36,36 \%)$.

La importancia de la interacción oral, decididamente, resulta manifiesta respecto a este ítem. Conforme a la opinión de los participantes, todas las destrezas son importantes en el aprendizaje de la lengua y se debe procurar práctica en cada una de ellas, pero la interacción oral, específicamente, es fundamental. Este es el único ítem, además, en el que hacen referencia explícita al currículo de la etapa, concretamente a la dimensión del alumno como «agente social», para recordar que «el aula es como una pequeña comunidad de personas; es importante que usen la lengua para comunicarse, no solamente para realizar y resolver tareas, como vimos en el MCERL. Si queremos transmitir la idea de que la lengua es para comunicarse y que es necesaria en el mundo real, tenemos que crear situaciones que los obliguen y los motiven a usarla, aunque sea en el contexto del aula, porque si no, estamos en un callejón sin salida, diciendo una cosa y haciendo otra».

\section{CONCLUSIONES DEL ESTUDIO}

La finalidad de este estudio consistió en identificar las creencias de los participantes sobre la enseñanza y aprendizaje del inglés como lengua extranjera antes de comenzar el periodo de prácticas en los centros, comprobar si sus respuestas acerca de esas creencias cambiaban al finalizarlo y, en tal caso, qué razones justificaban dichos cambios. Si se considera, de modo estricto, que los resultados obtenidos indican un único cambio con significatividad estadística, habría que concluir, entonces, que las experiencias vividas durante las prácticas no han influido apenas sobre esas creencias y que, por consiguiente, estas últimas son resistentes al cambio (Peacock, 2011).

Por otra parte, también es cierto que el sistema de creencias con el que cada participante comienza sus prácticas, al igual que el de cada uno de nosotros, está fuertemente enraizado y consolidado, resultado de muchos años de experiencias y vivencias (Numrich, 1996), tanto en contextos formales como no formales (Johnson, 1994), principalmente a lo largo de la enseńanza secundaria (Robertson, 1992). Ocho semanas de prácticas, evidentemente, no cambian un sistema de estas características.

Sin embargo, y aun aceptando la evidencia estadística, es necesario recordar que el análisis de grupos no refleja los cambios ni la evolución de las creencias individuales; pero esos cambios tienen lugar, como demuestran los resultados de este estudio, y, en todos los casos, las razones que los justifican -ya sea la escasa competencia en la lengua extranjera de los estudiantes de secundaria, la falta de motivación por aprenderla, la metodología de aula observada o las experiencias de aprendizaje propias-siempre responden a las discrepancias que surgen entre lo observado 
en el contexto real de la práctica y el sistema de creencias de los participantes respecto a lo que implica aprender y enseñar una lengua extranjera.

Las situaciones problemáticas o conflictivas de la práctica (Schön, 1998; Debreli, 2016), entonces, han permitido a los participantes tomar consciencia de sus propias creencias y reflexionar sobre las mismas, sobre su práctica y sobre la práctica profesional, y este proceso de toma de consciencia y de reflexión es el que, verdaderamente, puede generar cambios en las creencias de los futuros profesionales (Schön, 1998). «Las creencias [...] juegan un papel central en la adquisición e interpretación del conocimiento y la práctica docente subsiguiente [...] y pueden ser responsables de que prácticas anticuadas e ineficaces se perpetúen» (Pajares, 1992, p. 23).

Tomar consciencia de estas creencias es, sin duda, el primer paso para iniciar esos procesos de reflexión y cambio necesarios en el desarrollo y el ejercicio profesional, y los centros de secundaria proporcionan el contexto idóneo para llevarlo a cabo porque las prácticas externas, en cualquier programa de formación, constituyen la oportunidad de conocer y aprender la práctica profesional en el contexto profesional y real de la práctica; resulta crucial, entonces, proporcionar experiencias prácticas de mayor duración en los programas de formación (Busch, 2010; Çapan, 2014).

Por otro lado, es evidente, también, que la utilización de cuestionarios para identificar las creencias de estudiantes y profesores en fase de formación inicial es fundamental, ya que la información que aportan permite detectar carencias y concepciones erróneas que deberían ser abordadas en los cursos de formación.

Un ejemplo claro, en los resultados presentados en este estudio, fue la idea extendida de que aprender una lengua extranjera se reduce a conocer mucho vocabulario o que, de modo general, unas destrezas son más fáciles de aprender que otras. La falta de formación específica para trabajar la lengua extranjera con alumnos con necesidades educativas específicas, el modo y momento idóneos para corregir los errores que se cometen verbalmente o por escrito, o la manera adecuada de enfocar la evaluación y el trabajo específico con estrategias de aprendizaje son, igualmente, aspectos que surgieron a partir de las respuestas dadas a ítems relacionados con la dificultad de la lengua, las características del aprendizaje de las lenguas, o las estrategias para el aprendizaje y la comunicación, y lo observado durante las prácticas, aspectos todos que sería fundamental atender como parte integral de la formación inicial del profesorado de lenguas extranjeras.

Las creencias, definitivamente, no cambian de un día para otro, pero tampoco son inflexibles; cambian poco a poco, de manera gradual y acumulativa (Cabaroglu \& Roberts, 2000), a base de estímulos, contextos significativos y oportunidades de práctica; son, por tanto, resistentes, pero no impermeables al cambio. 


\section{REFERENCIAS BIBLIOGRÁFICAS}

Borg, S. (2003). «Teacher cognition in language teaching: a review of research on what language teachers think, know, belive and do». Language Teaching, (36), 81-109. Doi: 10.1017/ S0261444803001903.

Busch, D. (2010). «Pre-service teacher beliefs about language learning: the second language acquisition course as an agent for change». Language Teaching Research, 14(3), 318-337. Doi: 10. i i $77 / 1362$ i 688 i 0365239.

Cabaroglu, N. y Roberts, J. (2000). «Development in student teachers' pre-existing beliefs during a 1-year PGCE programme». System, (28), 387-402, recuperado de http://www.elsevier. com/locate/system.

Calderhead, J. y Robson, M. (1991). «Images of teaching: student teachers' early conceptions of classroom practice». Teaching and Teacher Education, 7(1), 1-8. Doi: 10.1016/0742-051X (91) 90053-R.

ÇAPAN, S.A. (2014). «Pre-service English as a foreign language teachers' belief development about grammar instruction». Australian Journal of Teacher Education, 39(12), 131-152. Doi: 10.14221/ajte.2014v39n12.9.

Debreli, E. (2016). «Pre-service teachers' beliefs change and practical knowledge development during the course of practicum». Journal of Education and Teaching Studies, 4 (7), 37-45. Doi: $10.11114 /$ jets.v.4i7.1513.

Finlay, L. (2008). Reflecting on 'reflective practice'. En Practice Based-Professional Learning Centre, The Open University, paper 52, recuperado de http://www.open.ac.uk./pbpl.

GAO, Y. (2014). «Language teacher beliefs and practices: a historical review». The Journal of English as an International Language, 9(2), 40-93, recuperado de http://www.researchgate.net/publication/269634374.

Horwitz, E.K. (1988). «The beliefs about language learning of beginning university foreign language students». The Modern Language Journal, 72(3), 283-294. Doi: 10.1111/j.15404781.1988.tb04190.X.

Horwitz, E.K. (1987). «Surveying student beliefs about language learning», en Wenden, A. y Rubin, J. (eds.). Learner strategies in language learning (119-129). New York, USA: Prentice Hall International.

Horwitz, E.K. (1985). «Using student beliefs about language learning and teaching in the foreign language methods course». Foreign Language Annals, 18(4), 333-340.

Johnson, K. (1994). «The emerging beliefs and instructional practices of preservice English as a second language teacher». Teaching and Teacher Education, 10(4), 439-452. Doi: 10.1016/0742051X (9) 900024-8.

Joram, E. y Gabriele, A.J. (1998). «Preservice teachers” prior beliefs: transforming obstacles into opportunities». Teaching and Teacher Education, 14(2), 175-191, recuperado de http://www. sciencedirect.com/science/article/pii/S0742-051X(97)00035-8.

KaGAN, D.M. (1992a). «Implications of research on teacher beliefs». Educational Psychologist, 27(1), 65-90. Doi: 10.1207/s15326985ep2701_6.

KaGan, D.M. (1992b). «Professional growth among preservice and beginning teachers». Review of Educational Research, 62(2), 129-169, recuperado de http://www.jstor.org/stable/1170578. 
Kuntz, P.S. (1996a). «Beliefs about language learning: the Horwitz model». ERIC Document Reproduction Service No. ED 397 649, Informe No. FL 023951.

Lightbown, P.M. y Spada, N. (2006). How languages are learned. Oxford, UK: OUP.

Mattheoudakis, M. (2007). «Tracking changes in pre-service EFL teacher beliefs in Greece: a longitudinal study». Teaching and Teacher Education, (23), 1272-1288. Doi: 10.1016/j. tate.2006.06.001.

Nikitina, L. y Furuoka, F. (2006). «Re-examining Horwitz's beliefs about language learning inventory (BALLI) in the Malaysian context». Electronic Journal of Foreign Language Teaching, 3(2), 209-219, recuperado de http://e-flt.nus.edu.sg/.

Numrich, C. (1996). «On becoming a language teacher: insights from diary studies». TESOL Quarteley, 30(1), 131-153, recuperado de http://www.jstor.org/stable/3587610.

ÖZmen, K.S. (2012). «Exploring student teachers' beliefs about language learning and teaching: a langitudinal study». Current Issues in Education, 15(1), 1-16, recuperado de https://cie.asu.edu.

Pajares, M.F. (1992). «Teachers' beliefs and educational research: cleaning up a messy construct». Review of Educational Reseach 62(3), 307-332, recuperado de http://www.jstor.org/stable/1170741.

Peacock, M. (2001). «Pre-service ESL teachers' beliefs about second language learning: a longitudinal study». System, (29), 177-195, recuperado de http://www.elsevier.com/locate/system.

Roberts, L.P. (1992). «Attitudes of entering university freshmen toward foreign language study: a descriptive analysis». Modern Language Journal, 76(3), 275-283, recuperado de http:// www.jstor.org/stable/330158.

Sснӧn, D.A. (1998). El profesional reflexivo. Cómo piensan los profesionales cuando actúan. Barcelona, España: Paidós Ibérica, S.A.

Wong, M.S. (2010). «Beliefs about language learning: a study of Malaysian pre-service teachers». RELC Journal, 41(2), 123-136. Doi: 10.1177/0033688210373124.

YuAn, R. y LeE, I. (2014). «Pre-service teachers' changing beliefs in the teaching practicum: three cases in an EFL context». System, (44), 1-12, recuperado de http://www.elsevier.com/ locate/system.

ZheNG, H. (2009). «A review of research on ESL pre-service teachers' beliefs and practices». Journal of Cambridge Studies, 4(1), 73-81, recuperado de http://journal.acs-cam.org.uk. 